\title{
Mortalidade por Câncer de Mama: uma Análise da Tendência no Ceará, Nordeste e Brasil de 2005 a 2015
}

doi: https://doi.org/10.32635/2176-9745.RBC.2020v66n1.740

\author{
Breast Cancer Mortality: a Trend Analysis in Ceará, Northeast and Brazil from 2005 to 2015 \\ Mortalidad del Cáncer de Mama: un Análisis de Tendencias en Ceará, Nordeste y Brasil de 2005 a 2015
}

\author{
Liana de Oliveira Barros'; Vanessa Barreto Bastos Menezes²; Antonia Cristina Jorge³; Sônia Sâmara Fonseca de Morais; Marcelo \\ Gurgel Carlos da Silva ${ }^{5}$
}

\section{Resumo}

Introdução: Informaçóes sobre mortalidade por câncer de mama podem ser úteis para o planejamento de políticas públicas. Objetivo: Analisar a tendência da mortalidade por câncer de mama em mulheres no Estado do Ceará. Método: Estudo descritivo exploratório cujas variáveis utilizadas foram: ano e local da ocorrência do óbito, sexo, causa básica de morte e a idade em faixa etária. A análise estatística dos dados foi realizada no programa Gretl, por meio de regressão linear, no qual as taxas de mortalidade por câncer de mama foram consideradas variáveis dependentes e os anos do período estudado, variáveis independentes. Foram apresentados os coeficientes de regressão, seus intervalos de confiança de $95 \%$ e os respectivos valores-p dos testes de significância estatística. Resultados: Foram notificados 141.168 óbitos por câncer de mama em todo o Brasil. O Estado Ceará representa 3,73\% desse total e apresentou um aumento de $92,7 \%$ no número de óbitos por câncer de mama. Observou-se um aumento das taxas específicas de mortalidade a partir dos 40 anos de idade se mantendo crescente até a última faixa etária pesquisada. Conclusáo: A análise realizada neste estudo evidenciou uma tendência progressiva no número de óbitos no Ceará, no Nordeste e no Brasil. É imprescindível a elaboração de estratégias que priorizem ações, a fim reduzir o atraso na condução dos casos de câncer de mama nos serviços de saúde para que a detecçáo precoce reduza o número de óbitos. Palavras-chave: Neoplasias da Mama/epidemiologia; Neoplasias da Mama/mortalidade; Brasil.

\section{Abstract}

Introduction: Information on breast cancer mortality can be useful for planning public policies. Objective: Analyze the trend of mortality from breast cancer in women in the state of Ceará. Method: Exploratory descriptive study whose variables were: year and place of death, sex, basic cause of death, and age in an age range. The statistical analysis of the data was performed in the Gretl program by means of linear regression where the breast cancer mortality rates were considered dependent variables and the years of the study period, the independent variables. The regression coefficients, their $95 \%$ confidence intervals and the respective p-values of the tests of statistical significance were presented. Results: 141,168 deaths from breast cancer were reported across Brazil. The state of Ceará represents $3.73 \%$ of this total and had a growth of $92.7 \%$ in the number of deaths from breast cancer. There was an increase in specific mortality rates from the age of 40 onwards, increasing until the last age group surveyed. Conclusion: The analysis carried out in this study showed a progressive trend in the number of deaths in Ceará, in the Northeast and in Brazil. It is essential to develop strategies that prioritize actions to shorten the delay in the management of breast cancer cases in health services so that early detection reduces the number of deaths.

Key words: Breast Neoplasms/epidemiology; Breast Neoplasms/mortality; Brazil.
Resumen

Introducción: la información sobre mortalidad por cáncer de mama puede ser útil para planificar políticas públicas. Objetivo: Analizar la tendencia de mortalidad por cáncer de seno en mujeres en el Estado de Ceará. Método: Estudio exploratorio descriptivo cuyas variables utilizadas fueron: ańo y lugar de muerte, sexo, causa básica de muerte y edad en un rango de edad. El análisis estadístico de los datos se realizó en el programa Gretl mediante regresión lineal donde las tasas de mortalidad por cáncer de mama se consideraron variables dependientes y los ańos del período estudiado fueron variables independientes. Se presentaron los coeficientes de regresión, sus intervalos de confianza del $95 \%$ y los respectivos valores p de las pruebas de significación estadística. Resultados: Se notificaron 141.168 muertes por cáncer de mama en todo Brasil. El Estado de Ceará representa el 3,73\% de este total e mostró un aumento del $92,7 \%$ en el número de muertes por cáncer de seno. Hubo un aumento en las tasas de mortalidad específicas a partir de los 40 años, aumentando hasta el último grupo de edad encuestado. Conclusión: El análisis realizado en este estudio mostró una tendencia progresiva en el número de muertes en Ceará, en el noreste y en Brasil. Es esencial desarrollar estrategias que prioricen las acciones para reducir la demora en el manejo de casos de cáncer de seno en los servicios de salud para que la detección temprana, reduciendo así el número de muertes. Palabras clave: Neoplasias de la Mama/epidemiología; Neoplasias de la Mama/mortalidad; Brasil.

\footnotetext{
1 Programa de Pós-Graduação em Saúde Coletiva (PPSAC), Centro de Ciências da Saúde (CCS), Universidade Estadual do Ceará (UECE). Fortaleza (CE), Brasil. Orcid iD: https://orcid.org/0000-0001-9935-8122

2 PPSAC/CCS/UECE. Fortaleza (CE), Brasil. Orcid iD: https://orcid.org/0000-0002-8951-6200

${ }^{3}$ Secretaria de Saúde do Estado do Ceará. Fortaleza (CE), Brasil. Orcid iD: https://orcid.org/0000-0002-0758-5676

${ }^{4}$ PPSAC/CCS/UECE. Fortaleza (CE), Brasil. Orcid iD: https://orcid.org/0000-0002-0146-4611

${ }_{5}^{5}$ PPSAC/CCS/UECE. Fortaleza (CE), Brasil. Orcid iD: https://orcid.org/0000-0003-4030-1206

Endereço para correspondência: Liana de Oliveira Barros. PPSAC/CCS/UECE. Fortaleza (CE), Brasil. Rua João Costa, 35 - Joaquim Távora. Fortaleza (CE), Brasil. CEP 60115-040. E-mail: lianabarross@gmail.com
} 


\section{INTRODUÇÃO}

O câncer de mama é considerado o mais prevalente entre mulheres. Em 2018, mais de dois milhôes de novos casos de câncer de mama surgiram em todo o mundo. Segundo o World Cancer Research Fund Internacional', em 2018, entre os países que apresentam maior incidência de câncer de mama estão a Bélgica, seguida por Luxemburgo e Holanda ${ }^{1}$.

A incidência de câncer de mama nos países desenvolvidos é maior, enquanto a mortalidade relativa é maior nos países menos desenvolvidos ${ }^{2}$. Um estudo sobre epidemiologia, incidência e mortalidade de câncer de mama na Ásia mencionou que o aumento em mortes por câncer de mama na Ásia é significativo em comparação com países europeus e americanos, sugerindo que essa Região possui um bom serviço informação de mortalidade ${ }^{3}$.

Para o Brasil, estimam-se 66.280 casos novos de câncer de mama a cada ano do triênio 2020-2022. Esse valor corresponde a um risco estimado de 61,61 casos novos a cada 100 mil mulheres. Sem considerar os tumores de pele náo melanoma, o câncer de mama feminina ocupa a primeira posição mais frequente em todas as Regiōes brasileiras, com um risco estimado de 81,06 por 100 mil na Região Sudeste; de 71,16 por 100 mil na Região Sul; de 45,24 por 100 mil na Região Centro-Oeste; de 44,29 por 100 mil na Região Nordeste; e de 21,34 por 100 mil na Região Norte ${ }^{4}$.

$\mathrm{O}$ acesso aos serviços de saúde no Brasil apresenta variaçóes entre as cidades no interior dos Estados e as suas respectivas capitais, que traduzem disparidades nas taxas de mortalidade por câncer de mama em todo o país 5 .

Em 2014, estudou-se o impacto do estádio da doença como preditor de prognóstico na sobrevida e concluiu-se que detectar a doença precocemente minimiza a mortalidade por câncer de mama. Os autores afirmam ainda que o câncer de mama, quando diagnosticado em fases iniciais, tem grandes chances de cura, com uma sobrevida de $97 \%$ em cinco anos ${ }^{6}$, embora, quando se considera o carcinoma in situ, haja ainda controvérsias na detecção precoce pelo diagnóstico de tumores indolentes.

Um estudo analisou 43.442 casos de câncer de mama, no período compreendido entre 1995 e 2002, revelando que $87,7 \%$ das mulheres diagnosticadas com câncer de mama encontravam-se entre os estágios II e IV. Essa realidade diverge dos países desenvolvidos, que, apesar de apresentarem maior prevalência da doença, têm um melhor sistema de detecção precoce, reduzindo os custos com o tratamento e a mortalidade entre as mulheres. A mediana do percentual de pacientes no Brasil entre os estágios II e IV é de 45,3\%, enquanto, nos Estados Unidos, é cerca de $12 \%{ }^{7,8}$.
Informações sobre incidência e mortalidade por câncer de mama podem ser úteis para o planejamento de políticas públicas e implementação de programas de controle de câncer baseados em evidências, porém, não se encontram disponíveis na maioria dos países de baixa e média rendas.

Nesse sentido, faz-se necessário analisar informações sobre o comportamento epidemiológico dos óbitos por essa patologia, permitindo o estabelecimento de estratégias de prevenção e promoção da saúde, com reduçấo dos gastos públicos com o tratamento da doença.

Além da morosidade do sistema de saúde, outros fatores estáo associados ao desenvolvimento da doença e mortalidade. Os fatores individuais têm um papel importante no câncer de mama, incluindo o estilo de vida (alimentação, atividade física e uso de drogas) e a constituição genética?.

Em 2018, um estudo encontrou resultados que demonstraram que a síndrome metabólica foi associada a um aumento de $73 \%$ no risco de mortalidade por câncer de mama. O mesmo estudo sugere que a circunferência da cintura alta, o colesterol alto e hipertensão foram independentemente associados ao aumento do risco de mortalidade por câncer de mama9?

Os fatores ambientais e socioeconômicos influenciam diretamente na problemática. Segundo o Ministério da Saúde, as formas mais eficazes de detecção precoce são o exame clínico da mama e a mamografia ${ }^{10}$.

A ultrassonografia pode ser realizada como apoio diagnóstico que, na maioria das vezes, prevê dados suficientes para estadiamento clínico do tumor e definição do tratamento. No entanto, muitas mulheres com perfil de risco não têm acesso a esses exames, pois esse acesso aos serviços de saúde em alguns locais do Brasil, incluindo o Ceará, ainda é restrito. Além disso, a mamografia no serviço público é realizada somente na faixa etária considerada "de risco", entre 50 e 59 anos ${ }^{11,12}$.

O objetivo deste estudo é analisar a tendência da mortalidade por câncer de mama em mulheres no Estado do Ceará, realizando comparativos entre os dados verificados no Nordeste e no Brasil entre os anos de 2005 a 2015.

\section{MÉTODO}

Trata-se de um estudo de dados agregados de uma série temporal de 11 anos da mortalidade por câncer de mama em pacientes do sexo feminino no Estado do Ceará, Regiāo Nordeste e Brasil no período de 2005 a 2015. Os dados sobre os óbitos foram extraídos da base de dados do Atlas On-line de Mortalidade por Câncer ${ }^{13}$, disponibilizados no site do Instituto Nacional de Câncer José Alencar Gomes da Silva (INCA) do Ministério da Saúde; e os dados demográficos, do Instituto Brasileiro de 
Geografia e Estatística (IBGE). O Atlas sobre mortalidade utilizado tem como uma de suas principais finalidades auxiliar os profissionais de saúde pública na determinação de prioridades necessárias à prevenção e ao controle do câncer ${ }^{13}$.

Os óbitos por câncer de mama para o período de 2005 a 2015 foram obtidos considerando a codificação (C50) da Classificaçấo Estatística Internacional de Doenças e Problemas Relacionados à Saúde - Décima Revisão (CID10). Foram utilizadas taxas de mortalidade por câncer de mama feminino, brutas e ajustadas por idade, pelas populaçôes mundial e brasileira, por 100 mil habitantes, faixa etária, localidade e por período. Foi selecionado somente o sexo feminino, que representa a maioria dos casos. As variáveis estudadas foram: ano e local da ocorrência do óbito, sexo, causa básica de morte e faixa etária. A idade foi agrupada nas seguintes faixas etárias: $(0$ a $4 ; 5$ a $9 ; 10$ a $14 ; 15$ a $19 ; 20$ a 29; 30 a 39; 40 a 49; 50 a 59; 60 a 69; 70 a 79; 80 anos ou + e idade ignorada).

Os dados utilizados foram inicialmente organizados no programa Excel para Windows e posteriormente analisados no programa Gretl ${ }^{14}$. Foram realizadas análises de tendência temporal por meio de regressão linear. Após verificação da inexistência de correlação entre os erros-padrão ao longo do tempo, por meio do teste de Breusch Godfrey, verificou-se a inexistência de autocorrelação para os casos do Brasil e Nordeste.

$\mathrm{Na}$ análise de regressão linear simples, as taxas de mortalidade por câncer de mama foram consideradas como variáveis dependentes (y) e os anos do período estudado como variável independente (x).
Com o objetivo de estimar o acréscimo ou decréscimo das taxas de mortalidade, foram apresentados os coeficientes de regressão, seus intervalos de confiança de $95 \%$ e os respectivos valores-p dos testes de significância estatística. Além disso, foi calculada a variaçáo anual percentual por meio da razão do coeficiente de regressão em relação à taxa de mortalidade no início do período analisado.

Os dados utilizados neste estudo são públicos e disponibilizados na base de dados do Atlas On-line de Mortalidade ${ }^{13}$ sem identificação dos indivíduos. Por essa razão, não houve a necessidade de encaminhamento do projeto para aprovação de um Comitê de Ética em Pesquisa.

\section{RESULTADOS}

No período estudado, foram notificados 141.168 óbitos por câncer de mama em todo o Brasil. Destes, 139.635 foram relativos ao sexo feminino, representando um percentual de $98,91 \%$ do total de casos. No Ceará, foram notificados 5.213 óbitos de pacientes do sexo feminino por câncer de mama, o que corresponde a 3,73\% do total, conforme demonstra a Tabela 1 .

No período compreendido entre 2010 e 2014, as taxas de mortalidade brutas do Ceará permaneceram relativamente estáveis, apresentando variaçóes mínimas entre os anos consecutivos. Já em 2015, o Ceará atingiu a maior taxa de mortalidade bruta $(14,05)$. Considerando todo o período estudado, ao chegar ao último ano, a taxa de mortalidade bruta desse Estado atingiu quase o dobro do valor quando comparada ao valor encontrado no primeiro ano analisado.

Tabela 1. Taxas de mortalidade por câncer de mama, brutas e ajustadas por idade, para as populações mundial e brasileira de 2010 , por 100 mil mulheres. Ceará, 2005-2015

\begin{tabular}{c|c|c|c|c}
\hline Ano & $\begin{array}{c}\text { Valor absoluto } \\
\text { (número de óbitos) }\end{array}$ & $\begin{array}{c}\text { Taxa de mortalidade } \\
\text { bruta - Ceará }\end{array}$ & $\begin{array}{c}\text { Taxa de mortalidade } \\
\text { ajustada mundial }\end{array}$ & $\begin{array}{c}\text { Taxa de mortalidade } \\
\text { ajustada brasil }\end{array}$ \\
\hline 2005 & 331 & 8,01 & 8,6 & 9,35 \\
2006 & 403 & 9,64 & 10,48 & 11,12 \\
2007 & 413 & 9,77 & 10,34 & 11,07 \\
2008 & 455 & 10,65 & 11,12 & 11,8 \\
2009 & 431 & 9,98 & 10,07 & 10,86 \\
2010 & 492 & 11,36 & 10,49 & 11,3 \\
2011 & 492 & 11,19 & 10,86 & 11,72 \\
2012 & 491 & 11,07 & 10,6 & 11,36 \\
2013 & 531 & 11,87 & 11,02 & 11,97 \\
2014 & 536 & 11,89 & 10,99 & 11,73 \\
2015 & 638 & 14,05 & 12,49 & 13,53 \\
Total & 5.213 & & & \\
\hline
\end{tabular}

Fontes: $\mathrm{IBGE}^{15} ; \mathrm{INCA}^{13}$. 
Destaca-se o período de 2010 a 2012, pois observou-se uma estabilidade no número de óbitos que variou entre 491 e 492 óbitos, tendo apenas uma discreta redução no último ano. Ao longo dos anos 2010 a 2012, verificou-se uma redução do valor das taxas brutas de mortalidade, voltando a crescer a partir de 2013. Outro fato que merece destaque é a diferença entre o primeiro ano analisado e o último, conforme observa-se na Figura 1.

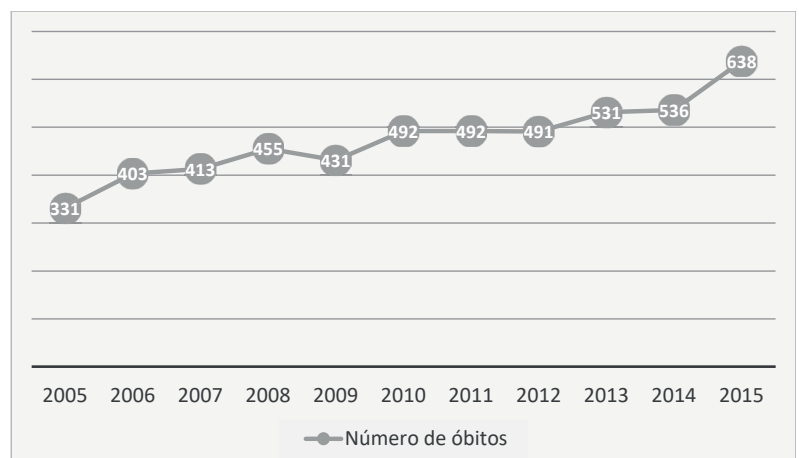

Figura 1. Tendência de óbitos por câncer de mama em homens e mulheres. Ceará, 2005-2015

Fonte: INCA ${ }^{13}$.
Em 11 anos, o Estado do Ceará apresentou um aumento significativo no número de mortes por câncer de mama. O número de óbitos de cearenses por esse tipo de câncer obteve aumento de $92 \%$ comparando o primeiro e o último ano analisado.

A Tabela 2 apresenta a tendência dos óbitos em mulheres no período estudado, bem como a tendência das taxas específicas de mortalidade por faixa etária, apresentando aumento consecutivo no número de óbitos até a faixa etária de 50-59 anos e reduzindo o número de óbitos a partir da faixa etária de 60-69 anos. Assim, observou-se que a Regiấo Nordeste e o Estado do Ceará repetiram o comportamento epidemiológico brasileiro.

Considerando o Brasil, o Nordeste e o Ceará na Tabela 2, conclui-se que as faixas etárias compreendidas entre 40 e 59 anos apresentaram os maiores números de óbitos, tendo uma redução a partir da faixa etária de 60 anos.

Em relação às taxas específicas de mortalidade por faixa etária, observou-se que, a partir da faixa etária 40-49 anos, ocorreu um aumento gradual destas no Brasil, que apresenta maiores valores de taxas, e o Ceará com taxas maiores do que as do Nordeste em todas as faixas etárias.

Tabela 2. Taxas de mortalidade por câncer de mama, brutas e ajustadas por idade, para as populações mundial e brasileira de 2010 , por 100 mil mulheres. Brasil, Nordeste e Ceará, 2005-2015

\begin{tabular}{|c|c|c|c|c|c|c|}
\hline \multirow[b]{2}{*}{$\begin{array}{l}\text { Faixa etária } \\
\text { (em anos) }\end{array}$} & \multicolumn{2}{|c|}{ Brasil } & \multicolumn{2}{|c|}{ Nordeste } & \multicolumn{2}{|c|}{ Ceará } \\
\hline & $\begin{array}{c}\text { Valor } \\
\text { absoluto } \\
\text { (número de } \\
\text { óbitos) }\end{array}$ & $\begin{array}{c}\text { Taxa } \\
\text { específica }\end{array}$ & $\begin{array}{c}\text { Valor } \\
\text { absoluto } \\
\text { (número de } \\
\text { óbitos) }\end{array}$ & $\begin{array}{c}\text { Taxa } \\
\text { específica }\end{array}$ & $\begin{array}{c}\text { Valor } \\
\text { absoluto } \\
\text { (número de } \\
\text { óbitos) }\end{array}$ & $\begin{array}{c}\text { Taxa } \\
\text { específica }\end{array}$ \\
\hline 0 a 4 & - & - & - & - & - & - \\
\hline 5 a 9 & - & 0 & 0 & 0 & - & - \\
\hline 10 a 14 & 1 & 0 & 1 & 0 & - & - \\
\hline 15 a 19 & 25 & 0,03 & 11 & 0,04 & 2 & 0,04 \\
\hline 20 a 29 & 1.135 & 0,6 & 294 & 0,53 & 56 & 0,65 \\
\hline 30 a 39 & 9.213 & 5,5 & 2.306 & 5,03 & 407 & 5,78 \\
\hline 40 a 49 & 24.378 & 17,45 & 5.530 & 15,68 & 988 & 17,56 \\
\hline 50 a 59 & 34.414 & 32,61 & 7.064 & 27,95 & 1213 & 30,29 \\
\hline 60 a 69 & 29.355 & 44,39 & 5.583 & 33,5 & 1059 & 39,3 \\
\hline 70 a 79 & 22.598 & 60,08 & 4.141 & 42,54 & 770 & 47,74 \\
\hline 80 ou mais & 18.499 & 100,22 & 3.563 & 74,05 & 717 & 88,33 \\
\hline Idade ignorada & 14 & 0 & 4 & 0 & 1 & 0 \\
\hline Total & 139.635 & & 28.498 & & 5.213 & \\
\hline Taxa bruta & & 12,88 & & 9,4 & & 10,9 \\
\hline $\begin{array}{l}\text { Taxa padrão } \\
\text { mundial }\end{array}$ & & 11,7 & & 9,45 & & 10,7 \\
\hline $\begin{array}{l}\text { Taxa padrão } \\
\text { Brasil }\end{array}$ & & 12,56 & & 10,16 & & 11,5 \\
\hline
\end{tabular}

Fontes: $\mathrm{IBGE}^{15}$; INCA ${ }^{13}$. 
Identifica-se um aumento de mais de oito vezes nas taxas específicas de mortalidade entre a faixa etária compreendida de 20-29 anos para a faixa de 30-39 anos. Triplicando na faixa seguinte e ainda com outro aumento para a posterior (50-59 anos), na qual há o maior quantitativo de pessoas acometidas.

A faixa etária de concentração dos óbitos no Ceará é facilmente identificada, ao se analisar a tendência no período de 2005 a 2015 na Tabela 2 . Nela, observa-se que o número de óbitos aumenta consideravelmente a partir de 40 anos, e que as maiores ocorrências foram na faixa etária de 50 a 59 anos e 60 a 69 anos.

Observou-se que 43,5\% dos óbitos por câncer de mama ocorreu em pessoas com faixa etária compreendida entre 50 a 69 anos de idade. Esses dados evidenciam que a grande maioria dos óbitos por câncer de mama ocorre nas pessoas com mais de 40 anos.

A tendência das taxas de mortalidade por câncer de mama em mulheres, no Ceará, seguiu a do Nordeste e do Brasil, apresentando crescimento gradual no período estudado.

A tendência de mortalidade no Ceará, no Nordeste e no Brasil apresentou, durante o período estudado, uma tendência ao aumento da mortalidade por câncer de mama. A análise dos resultados mostrou intervalo de confiança de $95 \%$. Foram consideradas significativas as variaçôes na taxa de mortalidade por câncer de mama que apresentaram nível de significância $\alpha=5 \%$ na regressão linear, conforme a Tabela 3.

\section{DISCUSSÃO}

Neste estudo, observou-se aumento das taxas bruta de mortalidade no Estado do Ceará entre os anos de 2005 a 2008, ocorrendo uma redução em 2009. Entre os anos de 2010 a 2014, essas taxas apresentaram valores semelhantes. Já no último ano, observou-se o maior aumento (18\%).

Esse comportamento epidemiológico difere em partes da tendência mundial, na qual, de acordo com os achados dos estudos realizados em diversos países, embora a prevalência da doença entre as mulheres continue alta, a mortalidade vem apontando para uma diminuiçãa ${ }^{16,17}$.
O aumento dessas taxas brutas de mortalidade no Estado do Ceará no último ano pesquisado pode ser justificado pelo aprimoramento de técnicas de informação de mortalidade nesse Estado, ou pela diminuição da mortalidade por causas mal definidas, e, sem dúvida, pelo crescimento populacional observado ao longo desse período.

A qualidade e a fidedignidade dos registros de óbitos encontrados no Ceará, no Brasil e demais países são fatores cruciais para fundamentar as informaçóes de mortalidade do câncer de mama.

No Brasil, o INCA, órgão do Ministério da Saúde, desempenha açóes de vigilância do câncer, com informaçôes obtidas por meio de Registros de Câncer e do Sistema de Informação sobre Mortalidade (SIM), centralizado na Secretaria de Vigilância à Saúde (SVS). A partir de 1999, foram criados, por meio das Secretarias Estaduais de Saúde, os núcleos técnicos de registro de câncer ${ }^{10}$.

É prudente considerar que a qualidade dos registros de óbitos dos Estados brasileiros não acontece de forma semelhante. $\mathrm{O}$ atraso na alimentação dos dados nos núcleos estaduais de registro de câncer pode comprometer a veracidade das informaçóes.

O aumento das taxas brutas de mortalidade no Ceará encontradas neste estudo pode estar relacionado ao fato de o serviço de registro de mortalidade por câncer de mama nesse Estado ser um dos que operam há mais tempo no Brasil, desde 1990, sempre participando de formaçóes, visando à qualidade das informaçóes registradas, podendo ser considerado um dos mais eficientes do país, principalmente quando comparado aos demais Estados do Nordeste brasileiro.

Embora tal sistema venha se aprimorando, aumentando sua cobertura e melhorando a qualidade de seus dados, ainda se encontram registros de óbitos por causas mal definidas, o que pode comprometer a análise da mortalidade, especialmente aquelas por causas específicas ${ }^{18}$.

Uma pesquisa de 2017 evidenciou que, no período de 2004 a 2014, as taxas de mortalidade por câncer de mama no Brasil (padronizadas por idade), a incidência de internação hospitalar e os custos com saúde pública

Tabela 3. Coeficientes de regressão, índices de confiança, valores-p e tendência das taxas de mortalidade de mulheres por câncer de mama, 2005-2015

\begin{tabular}{c|c|c|c|c}
\hline Região & IC 95\%* & Coeficiente & Valor-p & Tendência \\
\hline Brasil & 0,$35 ; 0,41$ & 0,37 & $<0,001$ & Aumento \\
Nordeste & 0,$36 ; 0,48$ & 0,42 & $<0,001$ & Aumento \\
Ceará & 0,$29 ; 0,57$ & 0,43 & $<0,001$ & Aumento \\
\hline
\end{tabular}

Legenda: ${ }^{*}$ IC $95 \%=$ intervalo de confiança de $95 \%$. 
aumentaram. Os autores encontraram ainda uma correlação positiva entre a doença e os custos públicos com saúde, constatando que a implementação de estratégias governamentais é eficaz contra o ônus desse câncer no Brasi $^{19}$. Essas estratégias podem ter influência direta na redução no número de óbitos por câncer de mama.

É importante ressaltar que os estudos de tendências mundiais indicam uma redução da mortalidade por câncer de mama nos próximos anos. O maior estudo já realizado no mundo analisou a tendência em 20 países. O resultado apresentou tendências favoráveis na América do Norte e Oceania, com previsão de uma redução adicional de $10 \%$ em suas taxas globais para 2020 , com a finalidade de atingir valores de 11-12/100 mil mulheres; ou seja, cerca de $50 \%$ mais baixas do que $1980^{20,21}$. Esses resultados podem ter relaçáo com o aumento na qualidade dos registros de óbitos por câncer de mama que justificam a criação de possíveis políticas de incentivo ao diagnóstico precoce da doença podendo ocasionar, assim, a redução da mortalidade.

A América Latina, o Brasil, o México, o Chile e a Colômbia apresentaram taxas estáveis, abaixo ou em torno de 10/100 mil. A mortalidade foi maior na Argentina, Cuba e Venezuela, no entanto, na Argentina, as tendências foram favoráveis nos últimos anos, com previsōes para 2020 , em torno de $16 / 100 \mathrm{mil}^{20,21}$.

Países como Hong Kong, Japão e Coreia apresentaram baixos índices de mortalidade, porém com tendências estáveis ou crescentes, com suas taxas permanecendo sempre abaixo de 10/100 mil. Enquanto, em Israel, Nova Zelândia e nas Filipinas, as taxas de mortalidade foram cerca de 16/100 mil habitantes em 2012. Entre os países analisados, as Filipinas apresentaram tendências sempre crescentes ${ }^{21}$.

É importante destacar que, mesmo após o diagnóstico, a demora em iniciar o tratamento reduz as chances de cura. A maioria das mulheres (57\%) aguarda de oito a 12 meses para iniciar o tratamento ${ }^{16,22}$. Há um maior percentual de mulheres com estágios III e IV. O estágio III ocorre em um terço das brasileiras admitidas em serviços de oncologia com câncer de mama ${ }^{19,23,24}$.

Um estudo mostrou forte associação entre o intervalo de tempo e a suspeita e a confirmação diagnóstica com o estadiamento ao diagnóstico do câncer ( $R P$ ajustada $=2,97$ e 3,04). Esse intervalo foi maior que seis meses em quase metade das mulheres $(42,7 \%)$, apontando, no período estudado, a morosidade do sistema de saúde ${ }^{21,25-27}$. A eficiência e eficácia dos serviços de saúde são determinantes para o sucesso da cura e reduçáo da mortalidade no Brasil.

Os fatores ambientais e socioeconômicos também influenciam diretamente na problemática. Segundo o Ministério da Saúde, as formas mais eficazes de detecção precoce são o exame clínico da mama e a mamografia ${ }^{10}$. A ultrassonografia pode ser realizada como apoio diagnóstico que, na maioria das vezes, prevê dados suficientes para o estadiamento clínico do tumor e a definição do tratamento.

No entanto, muitas mulheres com perfil de risco não têm acesso a esses exames, pois esse acesso aos serviços de saúde em alguns locais do Brasil, incluindo o Ceará, ainda é restrito. Sem falar que a mamografia no serviço público é realizada somente na faixa etária considerada "de risco", entre 50 e $59 \operatorname{anos}^{12,16,26}$. Neste estudo, mais de $90 \%$ dos óbitos ocorreram em mulheres com idade acima de 40 anos. Corroborando os achados desse estudo, em 2017, um estudo evidenciou que mais de $85 \%$ dos casos ocorrem após os 40 anos, alcançando seu pico dos 65 aos $70 \operatorname{anos}^{16}$.

Houve um aumento nas taxas de câncer de mama em mulheres mais jovens no Brasil, no entanto, a faixa etária entre 40 e 50 anos representa $74 \%$ dos casos $^{12}$. Considerando essa realidade, a Sociedade Brasileira de Mastologia ${ }^{28}$ recomenda a realizaçáo da mamografia anualmente a partir dos 40 anos. O Ministério da Saúde diverge dessa conduta, indicando que o exame seja feito a cada dois anos por mulheres com 50 anos ou mais (grupo considerado prioritário $)^{10}$.

Os resultados encontrados neste estudo devem ser analisados com prudência, uma vez que podem estar influenciados pela qualidade do diagnóstico, pelas informaçôes de óbito e pelo acesso a serviços de oncologia, fatores inerentes a cada Estado, Região ou país.

As taxas de mortalidade no Ceará poderão ser reduzidas conforme haja aumento do investimento do Estado em políticas públicas voltadas para a prevenção e controle da doença, por meio da estruturação das redes de atenção à saúde com inclusão de estratégias de promoção da saúde e prevenção de doenças.

Estudos de base mundial apontam para uma tendência à reduçáo da mortalidade por câncer de mama no Brasil em 2020. Porém, para que isso seja concretizado, faz-se necessário o investimento em políticas públicas no âmbito da saúde da mulher, que priorizam a identificação dos fatores de risco associados à doença, bem como o estabelecimento de estratégias que incluam a busca ativa dos casos e o diagnóstico precoce, para, então, reduzir o número de óbitos por câncer de mama.

\section{CONCLUSÃO}

A análise realizada neste estudo evidenciou uma tendência crescente no número de óbitos no Ceará, no Nordeste e no Brasil, embora, ao longo do período, tenha se observado sensíveis reduções.

É imprescindível a elaboração de estratégias que priorizem açôes que reduzam o atraso na condução dos 
casos de câncer de mama nos serviços públicos, para que a detecçáo precoce e, em consequência, a cura sejam realidades concretas no país, na Regiáo Nordeste e mais especificamente no Estado do Ceará.

O conhecimento dos fatores de risco, a prevenção e o diagnóstico precoce passam a ser vitais para a cura e a reabilitação das mulheres, de maneira que reduzam a mortalidade por câncer de mama.

Nesse sentido, a educação permanente dos profissionais que atuam no Sistema Único de Saúde (SUS) é imprescindível para o controle da doença, sendo necessário o desenvolvimento de competências que fortaleçam a educação em saúde e a capacidade de resolução de problemas, incluindo a busca ativa de mulheres com fatores de risco determinantes, bem como a melhoria da qualidade das informaçóes de mortalidade por parte dos estados.

Desse modo, é importante conhecer o perfil socioeconômico e demográfico das mulheres acometidas por tumores de mama, a fim de definir açóes assertivas que considerem as necessidades individuais e coletivas, visto que as crenças e os valores religiosos e culturais podem influenciar diretamente na detecção e tratamento delas.

\section{CONTRIBUIÇÕES}

Todos os autores contribuíram substancialmente na concepção e no planejamento do estudo, na obtenção, análise e/ ou interpretação dos dados, assim como na redação e/ou revisão crítica e aprovaram a versão final a ser publicada.

\section{DECLARAÇÃO DE CONFLITO DE INTERESSES}

Nada a declarar.

\section{FONTES DE FINANCIAMENTO}

Não há.

\section{REFERÊNCIAS}

1. Bray F, Ferlay J, Soerjomaram I, et al. Global cancer statistics 2018: GLOBOCAN estimates of incidence and mortality worldwide for 36 cancers in 185 countries. CA Cancer J Clin. 2018;68(6):394-424. doi: https://doi. org/10.3322/caac.21492

2. Ghoncheh M, Pournamdar Z, Salehiniya H. Incidence and mortality and epidemiology of breast cancer in the world. Asian Pac J Cancer Prev. 2016;17(S3):43-6. doi: https://doi.org/10.7314/apjcp.2016.17.s3.43

3. Ghoncheh M, Mahdavifar N, Darvishi F, et al. Epidemiology, incidence and mortality of breast cancer in Asia. Asian Pac J Cancer Prev. 2016;17(S3):47-52. doi: https://doi.org/10.7314/APJCP.2016.17.S3.47

4. Instituto Nacional de Câncer José Alencar Gomes da Silva. Estimativa 2020: incidência de câncer no Brasil. Rio de Janeiro: INCA; 2019.

5. Gonçalves LLC, Travassos GL, Almeida AM, et al. Barreiras na atenção em saúde ao câncer de mama: percepção de mulheres. Rev Esc Enferm USP. 2014;48(3):394-400. doi: https://doi.org/10.1590/ S0080-623420140000300002

6. Hofelmann DA, Anjos JC, Ayala AL. Sobrevida em dez anos e fatores prognósticos em mulheres com câncer de mama em Joinville, Santa Catarina, Brasil. Cien Saude Coletiva. 2014;19(6):1813-24. doi: https://doi. org/10.1590/1413-81232014196.03062013.

7. Coughlin SS, Ekwueme DU. Breast cancer as a global health concern. Cancer Epidemiol. 2009;33(5):315-18. doi: https://doi.org/10.1016/j.canep.2009.10.003

8. Cuzick J, DeCensi A, Arun B, et al. Preventive therapy for breast cancer: a consensus statement. Lancet Oncol. 2011;12(5):496-503. doi: https://doi.org/10.1016/ S1470-2045(11)70030-4

9. Rosato V, Bosetti C, Negri E, et al. Reproductive and hormonal factors, family history, and breast cancer according to the hormonal receptor status. Eur J Cancer Prev. 2014;23(5):412-7. doi: https://doi.org/10.1097/ CEJ.0b013e3283639f7a

10. Instituto Nacional de Câncer José Alencar Gomes da Silva. Diretrizes para a detecçáo precoce do câncer de mama no Brasil. Rio de Janeiro: INCA; 2015.

11. Wild CP, Weiderpass E, Stewart BW, editors. World Cancer Report: cancer research for cancer prevention. Lyon: IARC Press; 2018.

12. Dibaba DT, Ogunsina K, Braithwaite D, et al. Metabolic syndrome and risk of breast cancer mortality by menopause, obesity, and subtype. Breast Cancer Res Treat. 2018;174(1):209-18. doi: https://doi. org/10.1007/s10549-018-5056-8

13. Instituto Nacional de Câncer José Alencar Gomes da Silva. Atlas on-line de mortalidade [Internet]. Rio de Janeiro: INCA; c1996-2014. [acesso 2018 jul. 10]. Disponível em: https://www.inca.gov.br/app/mortalidade

14. Baiocchi G, Distaso W. GRETL: econometric software for the GNU generation. J Appl Econ. 2003;18(1):105110. doi: https://doi.org/10.1002/jae.704

15. Instituto Brasileiro de Geografia e Estatística [Internet]. Rio de Janeiro: IBGE; [acesso 2018 jul. 11]. Estimativas da população [1 tela]. Disponível em: https://www.ibge. gov.br/estatisticas/sociais/populacao/9103-estimativasde-populacao.html?edicao $=17283$

16. Souza NHA, Falcão LMN, Nour GFA, et al. Câncer de mama em mulheres jovens: estudo epidemiológico no nordeste brasileiro. SANARE. 2017;16(2):60-67. doi: https://doi.org/10.36925/sanare.v16i2.1179 
17. EBCTCG (Early Breast Cancer Trialists' Collaborative Group), McGale P, Taylor C, et al. Effect of radiotherapy after mastectomy and axillary surgery on 10-year recurrence and 20-year breast cancer mortality: metaanalysis of individual patient data for 8135 women in 22 randomised trials. Lancet. 2014;383(9935):2127-35. doi: https://doi.org/10.1016/S0140-6736(14)60488-8

18. França E, Teixeira R, Ishitani L, et al. Causas mal definidas de óbito no Brasil: método de redistribuição baseado na investigação do óbito. Rev Saúde Pública. 2014;48(4):671-681. doi: https://doi.org/10.1590/ S0034-8910.2014048005146

19. Figueiredo FWS, Almeida TCC, Cardial DT, et al. The role of health policy in the burden of breast cancer in Brazil. BMC Womens Health. 2017;17:121. doi: https:// doi.org/10.1186/s12905-017-0477-9

20. Carioli G, Malvezzi M, Rodriguez T, et al. Trends and predictions to 2020 in breast cancer mortality in Europe. Breast. 2017;36:89-95. doi: https://doi.org/10.1016/j. breast.2017.06.003

21. Carioli G, Malvezzi M, Rodriguez T, et al. Trends and predictions to 2020 in breast cancer mortality: Americas and Australasia. Breast. 2018;37:163-9. doi: https://doi. org/10.1016/j.breast.2017.12.004

22. Gonçalves PHB, Gaui MF, Martins RG, et al,. Padrão de tratamento cirúrgico do câncer de mama de acordo com a idade - Análise de 5 anos do Instituto Nacional do Câncer (INCA). Trabalho apresentado no XVI Congresso Brasileiro de Cancerologia e XIII Congresso Brasileiro de Oncologia Clínica, São Paulo, 26 a 30 de novembro de 2003.

23. Coleman MP, Quaresma M, Berrino F, et al. Cancer survival in five continents: a worldwide population-based study (CONCORD). Lancet Oncol. 2008;9(8):730-56. doi: https://doi.org/10.1016/S1470-2045(08)70179-7

24. Cuzick J. Preventive therapy for cancer. Lancet Oncol. 2017;18(8):PE472-PE482. doi: https://doi.org/10.1016/ S1470-2045(17)30536-3

25. Tarone RE. Birth cohort trends for breast cancer among women in Europe and North America. Epidemiol Biostat Public Health. 2017;14(1). doi: https://doi. org/10.2427/12306

26. Sant M, Allemani C, Capocacia R. Stage at diagnosis is a key explanation of differences in breast cancer survival across Europe. Int J Cancer. 2003;106(3):416-22. doi: https://doi.org/10.1002/ijc.11226

27. Soares PBM, Quirino Filho, Souza WP, et al. Características das mulheres com câncer de mama assistidas em serviços de referência do Norte de Minas Gerais. Rev Bras Epidemiol. 2012;15(3):595-604. doi: https://doi.org/10.1590/S1415-790X2012000300013

28. Sociedade Brasileira de Mastologia. Saiba tudo sobre o câncer de mama [Internet]. Rio de Janeiro: Sociedade Brasileira de Mastologia; [2016]. [acesso 2018 jul 10].
Disponível em: https://www.sbmastologia.com.br/wpcontent/uploads/2017/09/Cartilha-Saiba-Tudo-Sobreo-CM.pdf 